East African Medical Journal Vol. 87 No. 3 March 2010

SEXUAL BEHAVIOUR AMONG PERSONS LIVING WITH HIV / AIDS IN KAMPALA, UGANDA

D. Tumukunde, BSc, MPH, Nurse/Study Coordinator, Joint Clinical Research Centre, Makerere University School of Public Health, P.O. Box 7072, Kampala, Uganda, F. Nuwaha, MD, PhD, Associate Professor, E. Ekirapa, BDS, MPH, Assistant Lecturer, Makerere University School of Public Health, P.O. Box 7072, Kampala, Uganda, C. Kityo, MBChB, MSc, Deputy Director, F. Ssali, MBChB, MMed, Physician and P. Mugyenyi, MBChB, MMed, FRCP, Director, Joint Clinical Research Centre, Makerere University, School of Public Health, P.O. Box 7072, Kampala, Uganda

Requests for reprints to: Dr. F. Nuwaha, Makerere University, School of Public Health, P.O. Box 7072, Kampala, Uganda

\title{
SEXUAL BEHAVIOUR AMONG PERSONS LIVING WITH HIV/AIDS IN KAMPALA, UGANDA
}

\author{
D. TUMUKUNDE, F. NUWAHA, E. EKIRAPA, C. KITYO, F. SSALI and P. MUGYENYI
}

\begin{abstract}
Objective: To identify sexual behaviour and reproductive health needs of people living with HIV/AIDS (PLWHAs).

Design: A cross sectional study.

Setting: Joint Clinical Research Centre, Kampala Uganda.

Participants: Three hundred and eighty PLWHAs, 50\% of whom had initiated antiretro viral therapy (ART).

Main outcome measures: PLWHAs answered questions regarding sexual behaviour, number and type of sexual partners, symptoms of sexually transmitted infections, having been pregnant or causing a pregnancy, social demographic characteristics, consumption of alcohol, having biological children, desire for more children and use of condoms.

Results: In the past 12 months $227(60 \%)$ of the PLWHAs were sexually active. Of the sexually active $42(19 \%)$ never used condom, and $92(40 \%)$ used condoms inconsistently, thus 134 (35\%) of PLWHAs engaged in high risk sex. Two hundred and sixty five (70\%) said that PLWHAs can have healthy children and $115(30 \%)$ desired more children with $21(10 \%)$ of the women in the reproductive age group reporting a pregnancy and $22(17 \%)$ of the men reporting having caused a pregnancy. Only three $(7 \%)$ of the pregnancies were unplanned. Desire for more children was a strong independent predictor of engaging in high risk sex (Adjusted Odds Ratio 2.44, 95\% CI 1.35-4.42).

Conclusions: This study demonstrates that abstinence and use of condoms on their own may not be enough for HIV prevention among PLWHAs who desire children. Additional methods such as use of ART to reduce HIV infectiousness and sperm washing are needed.
\end{abstract}

\section{INTRODUCTION}

Most prevention strategies with the Human Immunodeficiency Virus (HIV) have been aimed at uninfected people to prevent them from becoming infected with HIV. People living with HIV/AIDS (PLWHAs) who are now estimated at 40 million globally are however important in prevention of HIV infection (1). The positive prevention strategy which aims to assist PLWHAs to take measures to avoid the possibility of exposing others to infection is currently of considerableinterest. The reasons for this interest are several. First PLWHAs have sex and a high proportion of them ranging from $10-60 \%$ continue to engage in unprotected sexual behaviours that place their partners at risk for infection and place themselves at risk for super infection with HIV and other sexually transmitted infections (STIs) (2). Second, the widespread use of anti-retroviral therapy (ART) improves the quality and sexual lives of PLWHAs. Although current evidence suggests that use of ART among HIV-positive individuals does not necessarily increase high risk sex among HIV-positive individuals (3), high risk sexual behaviour within the population among people who do not know their HIV status and among the HIV-negative is common (4). Third PLWHAs get to grips with the complex ethical issues such as disclosure, having children and minimising of transmission of HIV to their sexual partners as well as their children $(1,5)$. Fourth from an epidemiological and public health perspective, PLWHAs are deemed the most important group to address with prevention strategies for HIV and other sexually transmitted infections (STls). Preventive interventions with PLWHAs are likely to have a greater impact on the HIV epidemic, for an equivalent input of cost, time, 
resources, than preventive interventions focused on HIV negative individuals. A change in the high risk sexual behaviour of a HIV positive person will, on average and in almost all affected populations, have a much bigger effect on the spread of the virus than an equivalent change in the behaviour of a negative person (6). Sero-discordant couples are a particularly important group to support, as are infected pregnant women who are at risk of transmitting HIV to their infants (5). Finally from a human rights perspective, PLWHAs have a right to live well with HIV, which includes having a healthy sex life (1). This requires strategies that support people with HIV to protect their sexual health, to avoid new STls to delay HIV / AIDS disease progression and to protect PLWHAs HIV from re-infection or super-infection (7).

PLWHAshave specific prevention requirements that demand tailored communications or service delivery strategies. For instance, PLWHAs have the right to information to support their choices on whether to have children or not. The challenge is how to meet these specific needs without increasing stigma and discrimination. It has been stressed that involving PLWHAs in decision making for positive prevention is of paramount importance and probably the best way to approach positive prevention (8). Understanding the sexual behaviour and reproductive choices of PLWHAs is the first step towards greater involvement of PLWHAs in positive prevention $(1,8)$. More information is needed regarding sexual behaviour, reproductive choices and involvement of PLWHAs in positive prevention especially in low income countries of sub-Saharan Africa with the greatest burden of HIV / AIDS. This study aimed to identify sexual behaviour among PLWHAs in care in Kampala, the capital city of Uganda.

\section{MATERIALS AND METHODS}

The study was carried out in Kampala, Uganda at the Joint Clinical Research Centre (JCRC). The JCRC is a specialised medical institution for HIV/AIDS research and health care that was established in 1991. In 1996 the JCRC pioneered the use of ART in Uganda and has cumulatively provided anti-retroviral (ARV) drugs to more than 40,000 PLWHAs. More than 80\% of the PLWHAs attending for care at JCRC receive all services free of charge. HIV / AIDS patients who are not medically eligible for ART are provided basic health care including provision of cotrimoxazole prophylaxis, diagnosis and treatment of sexually transmitted infections, clinical screening for tuberculosis and prevention with positives strategy that includes provision of condoms and risk-reduction counselling. Referral is also provided to other health units for services not offered at the centre.

Design: In a cross section study, we interviewed a consecutive random sample of 190 PLWHAs who had initiated ART (ART experienced) and 190 PLWHAs who had not initiated ART (ART naive) attending for care at the JCRC from $5^{\text {th }}$ March to $4^{\text {th }}$ April 2007. The sample size was enough to detect the predictors of high risk sexual behaviour with a power of at least $80 \%$, a $95 \%$ confidence interval $(95 \% \mathrm{Cl})$ with an odds ratio of 2.5 if the prevalence of high risk sexual behaviours and of their predictors ranged between $10-80 \%$ in the study population. This sample was arrived at using epiinfo version 6 statcal for cross sectional studies using being ART naive or ART experienced as reference predictor variable. In order to be included for the interview, PLWHAs had to be in care for at least 12 months and be above 18 years of age. PLWHAs answered questions regarding sexual behaviour, number and type of sexual partners, symptoms of STls (urethral discharge, genital ulcer, low abdominal pain or vaginal discharge), having been pregnant or causing a pregnancy, social demographic characteristics, consumption of alcohol, having biological children, desire for more children and use of condoms. The dependent variable was engagement in high risk sex or not (high risk sex was defined as having had sex with a regular or non regular partner without a condom in the last 12 months).

Selection of study participants: We aimed to include equal number of ART experienced and ART naive PLWHAs because we needed adequate numbers to be able to analyse the effect of exposure to ART on high risk sexual behaviour. Systematic sampling was used to select participants for this study. Adult monthly attendance at the JCRC Kampala site is about 6840 HIV / AIDS patients (5696 ART experienced and 1144 ART naive). Of these, 4902 (ART experience) have taken ART and 994 (ART naive) were on prophylactic treatment for at least 12 months. Lists of PLWHAs are generated from the database separately for ART experienced or ART naive and these lists constitute the sampling frames. The PLWHAs come to JCRC for monthly reviews and drug refills and the clinic runs five days a week (Monday to Friday). In the database, each patient is given a code number. The database contains information for identification, records of treatment, date of initiation of care and dates of next visit. Daily attendance was 223 PLWHAs for ART experienced and 45 for the ART naive. We aimed to interview at least nine ART naive or experienced PLWHAs daily so every $25^{\text {th }}$ ART experienced and every $5^{\text {th }}$ ART naive patient was eligible for inclusion. Since the dates of review for each patient were on record, selected patients were interviewed on the specific dates of appointment in the outpatient clinic on a daily basis as they came in for their monthly scheduled visits. Patients who were selected but failed to report on their specific days, were tracked on subsequent days. Patients who refused the interview (about 2\%) were excluded. 
Data collection and analysis: Data was collected by trained research assistants supervised by one of the principal investigators(DT). Astandardised pre-tested interview schedule with both structured and semi-structured questions was used to collect the data. At the end of each day, data collected was sorted, checked for consistency and for completeness. After all data had been collected, it was entered into EpiData software version $2.1 \mathrm{~b}$ (EpiData Association, Odense, Denmark) and exported to STATA 8.2 (StataCorp LP) for analysis. The analysis included generation of frequencies, cross tabulations to investigate associations between various variables and engagement in high risk sex and binary logistic regression analysis to identify independent predictors of engagement in high risk sex. At the bivariate level, the associations of dichotomous variables wereassessed by use of chi square $\left(\mathrm{X}^{2}\right)$ or Fischer's exact test and by use of crude odds ratios (COR) and their corresponding $95 \%$ confidence interval $(95 \% \mathrm{Cl})$. Continuous variables were compared using F-ratios. All variables that were found significantly associated with high risky sexual behaviours from bivariate analysis were included in the binary logistic regression model using backward likelihood ratio method to generate a model to explain engaging in high risk sex. Before the logistic regression the variables were tested for multi-co linearity. The adjusted odds ratios (AOR) and the 95\% CI were then estimated for variables that remain significant after the logistic regression. In all the analysis a $\mathrm{P}<0.05$ was used as test for significance.

Ethical considerations: The study was approved by the Makerere University School of Public Health institutional review board and by the Uganda
National CouncilofScience and Technology. Informed consent was sought from each participant before the interview and confidentiality was ensured.

\section{RESULTS}

Of the 380 patients, $37 \%$ had been in care for less than 24 months, $40 \%$ for between 24 and 36 months whereas $23 \%$ had been in care for more than 36 months. About a third of the PLWHAs (127 of 380) were men with no difference in the percent of men between ART experienced and ART naïve groups ( $34 \%$ versus $33 \%)$. The age range was $18-73$ years with median age of 40 years and standard deviation of 10 years. Women were younger (mean age 36 years versus 41 years) than men (F-ratio 22.63,379 degrees of freedom [df], $\mathrm{P}<0.001)$. Most of the respondents $(53 \%)$ had attained secondary education, $35 \%$ were married, $35 \%$ were widowed, $48 \%$ unemployed and 35\% earned less than 30 United States dollars per month (about 50,000 Uganda shillings). Women (39\% versus 25\%) were more likely to be widowed than men $\left(X^{2}=6.66\right.$, $\mathrm{P}=0.01)$. The median CD4 count of the respondents was 317 cells $/ \mathrm{mm}^{3}$ with inter-quartile range (IQR) of 154 and ranges 61-853 cells $/ \mathrm{mm}^{3} 48(13 \%)$ had CD4 counts of less than 200,175 (48\%) had CD4 counts between 201 and 349 whereas $149(39 \%)$ had a CD4 count of more than 350 cells $/ \mathrm{mm}^{3}$ There were no differences between ART experienced and ART naïve regarding sex, educational level, religion, residence and occupation. However the ART experienced were more likely to be older, widowed and to have a higher income (Table 1).

Table 1

Socio-demographic characteristics among ART experienced and ART naive PLWHAs

\begin{tabular}{|c|c|c|c|c|c|c|}
\hline \multirow[t]{2}{*}{ Variable } & \multicolumn{2}{|c|}{ ART Experienced } & \multicolumn{2}{|l|}{ ART Naive } & \multirow[t]{3}{*}{$X^{2}(\mathrm{df})$} & \multirow[t]{3}{*}{ P-value } \\
\hline & Frequency & $(\%)$ & Frequency & $(\%)$ & & \\
\hline \multicolumn{5}{|l|}{ Sex } & & \\
\hline Male & 64 & 34 & 63 & 33 & & \\
\hline Female & 126 & 66 & 127 & 67 & $0.00(1)$ & 1.00 \\
\hline \multicolumn{7}{|l|}{ Age in years } \\
\hline $18-30$ & 25 & 13 & 70 & 37 & & \\
\hline $31-40$ & 93 & 49 & 82 & 43 & & \\
\hline $41-50$ & 64 & 34 & 22 & 12 & $45.19(3)$ & \\
\hline$\geq 50$ & 8 & 4 & 16 & 8 & & 0.001 \\
\hline \multicolumn{7}{|c|}{ Education level } \\
\hline None & 8 & 4 & 18 & 9 & & \\
\hline Primary & 59 & 32 & 55 & 29 & & \\
\hline Secondary & 92 & 48 & 91 & 48 & $4.43(3)$ & 0.21 \\
\hline Tertiary & 31 & 16 & 26 & 14 & & \\
\hline
\end{tabular}




\begin{tabular}{|c|c|c|c|c|c|c|}
\hline \multicolumn{7}{|l|}{ Religion } \\
\hline Catholic & 75 & 40 & 78 & 41 & \multirow{4}{*}{$6.52(3)$} & \multirow{4}{*}{0.09} \\
\hline Protestant & 55 & 29 & 68 & 36 & & \\
\hline Moslem & 23 & 12 & 24 & 12 & & \\
\hline Others & 37 & 19 & 20 & 11 & & \\
\hline \multicolumn{7}{|l|}{ Marital status } \\
\hline Single & 17 & 9 & 35 & 20 & \multirow{4}{*}{$11.50(3)$} & \\
\hline Married & 68 & 36 & 65 & 34 & & \\
\hline Divorced/Separated & 28 & 14 & 35 & 18 & & \\
\hline Widowed & 77 & 41 & 53 & 28 & & 0.009 \\
\hline \multicolumn{7}{|l|}{ Occupation } \\
\hline Unemployed & 48 & 25 & 49 & 26 & \multirow{4}{*}{$3.42(3)$} & \multirow{4}{*}{0.33} \\
\hline Peasant & 31 & 16 & 39 & 21 & & \\
\hline Business & 67 & 35 & 72 & 38 & & \\
\hline Civil servant & 43 & 24 & 30 & 16 & & \\
\hline \multicolumn{7}{|c|}{ Income (Uganda shillings per month)* } \\
\hline No income & 59 & 31 & 67 & 35 & & \\
\hline$<50,000$ & 66 & 35 & 67 & 35 & & \\
\hline $50,000-99,999$ & 10 & 5 & 21 & 11 & & \\
\hline $100,000-199,999$ & 18 & 9 & 15 & 8 & & \\
\hline $2000,000-299,999$ & 12 & 7 & 11 & 6 & \multicolumn{2}{|l|}{16.01} \\
\hline$\geq 300,000$ & 25 & 13 & 9 & 5 & (5) & 0.007 \\
\hline \multicolumn{7}{|l|}{ Residence } \\
\hline Rural & 88 & 46 & 77 & 41 & \multirow[t]{2}{*}{$1.07(1)$} & \multirow[t]{2}{*}{0.30} \\
\hline Urban & 102 & 54 & 113 & 59 & & \\
\hline
\end{tabular}

$\mathrm{df}$ are degrees of freedom

* 1 US Dollar $=1666$ Ugandan Shillings

Sexual behaviour within the past 12 months: Of the 380 study respondents $227(60 \%)$ reported sexual activity in the last 12 months. Of the sexually active $92(40.5 \%)$ were inconsistent users of condoms and $42(18.5 \%)$ never used a condom. Thus $134(35.3 \%)$ of the PLWHAs engaged in high risk sex (did not abstain, were inconsistent condom users or never used a condom). The ART naive 79/190 were more likely to engagein high risk sex than ART experienced $55 / 190\left(x^{2}=6.10, P=0.013\right)$. Women ( $49 \%$ versus $24 \%$ ) were more likely to abstain from sex compared to men $\left(x^{2}=21.57, P<0.001\right)$. However, there was no difference between men and women $(51 \%$ versus $49 \%$ ) regarding the consistency of condom use.

Of the 380 respondents, $89(23 \%)$ reported at least one symptom of an STI. Among the men, the reported symptoms were urethral discharge by 20 (16\%), genital ulcer by $16(13 \%)$ and other symptoms by $10(8 \%)$. Among the women, $54(21 \%)$ reported vaginal discharge, $31(12 \%)$ reported genital ulcers and $15(6 \%)$ reported low abdominal pain.
Twenty one $(10 \%)$ of the women in the reproductive age group reported having been pregnant, and $22(17 \%)$ of the men reported having impregnated someone. Only three $(7 \%)$ of the pregnancies (oneamong women and two among those caused by men) were reported to be un-wanted or unplanned. Of all the respondents, 115 (30\%) desired more children and $265(70 \%)$ said that an HIV positive individual can have healthy children.

As seen in Table 2, the ART experienced were more likely than the ART naive to have used condoms consistently, know the spouse or regulars partner's HIV status, but less likely to have never used or used condoms inconsistently. On the other hand there were no differences between ART experienced and ART naive patients regarding being sexually active, having had sex with a non-regular partner, having disclosed HIV status to spouse or regular partner, reporting of STIsymptoms, being pregnant or causing a pregnancy, desiring more children and saying that PLWHAs can have healthy children. 
Table 2

Sex and reproductive behaviour in the past 12 months among ART experienced and ART naïve PLWHAs

\begin{tabular}{|c|c|c|c|c|c|c|}
\hline Variable & $\begin{array}{l}\Gamma \exp \\
\text { No. }\end{array}$ & $\begin{array}{l}\text { erienced } \\
(\%)\end{array}$ & $\begin{array}{r}\text { ART } \\
\text { No. }\end{array}$ & $\begin{array}{c}\text { Naive } \\
(\%) \\
\end{array}$ & $X^{2}(\mathrm{df})$ & P-value \\
\hline \multicolumn{7}{|l|}{ Sexual activity in previous 12 months } \\
\hline Sexually active & 110 & 58 & 117 & 62 & $0.39(1)$ & 0.530 \\
\hline Sex with non-regular partners & 42 & 38 & 48 & 41 & $0.36(1)$ & 0.546 \\
\hline \multicolumn{7}{|l|}{ Condom use among the sexually active $(n=227)$} \\
\hline Consistent & 55 & 56 & 38 & 43 & & \\
\hline Inconsistent & 40 & 44 & 52 & 57 & & \\
\hline Never & 15 & 14 & 27 & 23 & $7.89(2)$ & 0.019 \\
\hline \multicolumn{7}{|c|}{ Disclosed HIV status to spouse/regular partner $n=227$} \\
\hline & 94 & 85 & 90 & 77 & $5.34(1)$ & 0.21 \\
\hline \multicolumn{7}{|c|}{ Know spouse or regular partner's HIV status $(n=227)$} \\
\hline & 92 & 83 & 66 & 56 & $24.77(1)$ & $<0.001$ \\
\hline \multicolumn{7}{|c|}{ Consequences of engaging in high risk sexual behaviour $(n=380)$} \\
\hline Reported STI symptoms & 45 & 24 & 44 & 23 & $0.00(1)$ & 1.00 \\
\hline Became pregnant (women $<50$ years) & 13 & 12 & 8 & 8 & $0.69(1)$ & 0.408 \\
\hline Caused pregnancy (men) & 11 & 16 & 11 & 19 & $0.05(1)$ & 0.831 \\
\hline \multicolumn{7}{|c|}{ Reproductive health desires and beliefs $(n=380)$} \\
\hline Desire more children & 55 & 29 & 60 & 32 & $0.20(1)$ & 0.655 \\
\hline HIV + can have healthy children & 137 & 72 & 128 & 67 & $0.80(1)$ & 0.372 \\
\hline
\end{tabular}

For column four numbers in brackets are degrees of freedom (df)

Riskfactors for engaging in high risk sexual behaviour: The results of the bivariate analysis are shown in Tables 3 and 4. Table 3 shows that being:ART naïve, 31-50years, male, married, employed, a Muslim, in care for $<24$ months and drinking of alcohol increased the chance of engagement in high risk sexual behaviour.

Table 3

Socio-economic factors associated with high risk sexual behaviour in the last 12 months among PLWHAs with corresponding crude odds ratios (COR) and 95\% confidence intervals (95\% CI)

\begin{tabular}{|c|c|c|c|c|}
\hline Variable & $\begin{array}{c}\text { Low / No risk } \\
(\mathrm{n}=134)\end{array}$ & $\begin{array}{l}\text { High risk } \\
(\mathrm{n}=246)\end{array}$ & COR & $95 \% \mathrm{CI}$ \\
\hline \multicolumn{5}{|l|}{ ART experience } \\
\hline Experienced & 55 & 135 & 1.0 & \\
\hline Naive & 79 & 111 & 1.75 & $1.12-2.74$ \\
\hline \multicolumn{5}{|l|}{ Age-group (years) } \\
\hline$>50$ & 15 & 68 & 1.0 & \\
\hline $41-50$ & 50 & 97 & 2.34 & $1.16-4.75$ \\
\hline $31-40$ & 61 & 62 & 4.46 & $2.20-9.14$ \\
\hline$<30$ & 8 & 19 & 1.91 & $0.63-5.75$ \\
\hline \multicolumn{5}{|l|}{ Sex } \\
\hline Female & 80 & 173 & 1.0 & \\
\hline Male & 54 & 73 & 1.60 & $1.01-2.55$ \\
\hline \multicolumn{5}{|c|}{ CD4 Count (cells $/ \mathrm{mm}^{3}$ ) } \\
\hline 200 and below & 18 & 30 & 1.0 & \\
\hline $201-349$ & 62 & 121 & 0.85 & $0.42-1.74$ \\
\hline 350 and above & 54 & 95 & 0.95 & $0.46-1.96$ \\
\hline
\end{tabular}


Marital status

$\begin{array}{lcccc}\text { Married } & 73 & 60 & 3.71 & 2.31-5.95 \\ \text { Not married } & 61 & 186 & 1.0 & \end{array}$

Education status

Some

127

227

1.52

$0.58-4.10$

None

7

19

1.0

Employment status

Employed 85

85

128

1.60

$1.02-2.52$

Not employed

49

118

1.0

Monthly income (Uganda shillings)

More than 50,000 51

50,000 and below 83

70

1.54

$0.97-2.47$

Religious affiliation

$\begin{array}{lc}\text { Muslem } & 26 \\ \text { Christian } & 108\end{array}$

176

1.0

Duration of care (months)

$\begin{array}{lcccc}<24 & 77 & 65 & 3.67 & 1.95-6.94 \\ 25-36 & 48 & 104 & 1.43 & 0.75-2.72 \\ >36 & 21 & 65 & 1.0 & \end{array}$

Drinks alcohol

\begin{tabular}{ccccc} 
Yes & 48 & 51 & 2.13 & $1.30-3.50$ \\
No & 86 & 195 & 1.0 & \\
\hline
\end{tabular}

*1 US Dollar $=1666$ Ugandan Shillings

Table 4

Association between engagement in high risk sexual behaviour with desire for children and beliefs towards children and use of condoms, and corresponding crude odds ratios (COR) with their 95\% confidence interval (95\% CI)

\begin{tabular}{|c|c|c|c|c|}
\hline Variable & $\begin{array}{c}\text { Low / No risk } \\
(\mathrm{n}=134)\end{array}$ & $\begin{array}{c}\text { High risk } \\
(\mathrm{n}=246)\end{array}$ & COR & $95 \% \mathrm{CI}$ \\
\hline \multicolumn{5}{|c|}{ Desires more children } \\
\hline Yes & 55 & 60 & 2.16 & $1.34-3.47$ \\
\hline No & 79 & 186 & 1.0 & \\
\hline \multicolumn{5}{|c|}{ Has biological children } \\
\hline Yes & 126 & 221 & 1.78 & $0.74-4.44$ \\
\hline No & 8 & 25 & 1.0 & \\
\hline \multicolumn{5}{|c|}{ PLWHAs can have healthy children } \\
\hline Agree & 96 & 168 & 1.17 & $0.72-1.91$ \\
\hline Disagree & 38 & 78 & 1.0 & \\
\hline \multicolumn{5}{|c|}{ Condoms reduce sexual pleasure } \\
\hline Agree & 52 & 52 & 2.37 & $1.45-3.86$ \\
\hline Disagree & 82 & 194 & 1.0 & \\
\hline \multicolumn{5}{|c|}{ Condoms not suitable for some sexual styles } \\
\hline Agree & 43 & 53 & 1.72 & $1.04-2.84$ \\
\hline Disagree & 91 & 193 & 1.0 & \\
\hline \multicolumn{5}{|c|}{ No need to use condoms if HIV positive } \\
\hline Agree & 13 & 15 & 1.65 & $0.71-3.82$ \\
\hline Disagree & 121 & 231 & 1.0 & \\
\hline
\end{tabular}


Education status, monthly income and CD4 cell count appeared not to influence engagement in high risk sexual behaviour. As seen in Table 4 engagement in high risk sexual behaviour was increased by having desired more children, agreeing that condoms reduced sexual pleasure and saying that condoms were not suitable for some sexual styles. On the other hand, having biological children saying that HIV positive people needed not to use condoms and agreeing that PLWHAs could have healthy children did not influence engagement in high risk sexual behaviour.

Independent predictors of engaging in high risksexual behaviour. The independent predictors of engaging in high risk sex included: being between 31 and 50 years of age (AOR 2.21,95\% CI 1.12-4.14), being married (AOR 3.91, 95\% CI 2.33-6.56), drinking of alcohol AOR 1.89, 95\% CI 1.08-3.34, desiring more children (AOR 2.44 95\% CI 1.35 - 4.42) and saying that condoms reduced sexual pleasure AOR 2.29, 95\% CI 1.30-4.05).

The model with these five variables was statistically reliable (goodness of fit $\mathrm{X}^{2}=13.04,8 \mathrm{df}$, $\mathrm{P}=0.11$ ), indicating that the predictors, as a set, reliably distinguished between PLWHAs who engaged in high risk sexual behaviours and those who did not. Variables that were significant on bivariate analysis but did not reach significance on binary logistic regression were: sex, religion, ART experience, employment status, duration in care, and saying that condoms are not suitable for some sexual styles.

\section{DISCUSSION}

This study found that about one in three of PLWHAs engaged in high risk sexual behaviours and that about $23 \%$ of PLWHAs reported symptoms of an STI. These findings are particularly worrying for transmission of HIV. Having an STI can increase the risk of passing HIV onto a partner through sex (9). Although there is growing evidence that people with HIV get reinfected and or super-infection with another 'strain' of HIV, including drug-resistant strains (10), this is thought not to be of major public health significance $(3,11)$. There is also increased chance that those who become pregnant are likely to transmit the HIV by mother to child transmission (MTCT) (11).

Our data is in agreement with previous research that use of ART at individual level does not necessarily increase high risk sex $(3,12)$. Moreover ART by diminishing the viral load in an individual on treatment greatly decreases the probability of sexual as well as mother to child transmission of HIV (11).

However these advantages of ART at individual level are not necessarily replicated at population level. More than $80 \%$ of the people who are HIV infected in Uganda and other low income countries have never had HCT and since they do not know their
HIV status they are not motivated to use protective measures (13). More so, only $20 \%$ of the PLWHAs who are eligible for ART in low income countries are actually on treatment (14), meaning that the majority of PLWHAs do not benefit from the use of ART including the reduced transmission of HIV to sexual partners. Furthermore, concerns that widespread availability of ART may cause dis-inhibition in the general population leading to increase in high risk sexual practices by removing the fear of death from AIDS are indeed still real (15).

Previous studies are also in consonance with our findings implicating use of alcohol (16) and being married (17) as a predictor of high risk sexual behaviour. Findings in this study pointing to the expressed need for PLWHAs to have children could explain why high risk sexual behaviour may be more common among married couples. Where the cultural pressure among married people is to have children as is the case in Uganda, condom use (which is a contraceptive) and sexual abstinence may be unrealistic as positive prevention strategies where conception is desired (17).

An explanation is warranted as to why PLWHAs aged 31-50 years are at increased chance of engaging in high risk sex. A possible reason is that people of this age group are at increased pressure to have a child (before 30 years, there is still time to have a child, and those above 50 years have probably already had one). However in this study both age and desire for children were tested in a multivariate logistic regression and both emerged significant. Thus if the effect of age was being mediated through desire for children, age would not be significant in the final regression model. Thus age and desire for children both being significant could be because PLWHAs in this study underreported their desire for children since this is the most socially desirable answer (18).

While interpreting this data it is important to know that this represents a highly selected population of PLWHAs who knew their HIV status and were in care in a centre of excellence like the joint clinical research centre. These data may therefore not be applicable to other PLWHAs who do not know their HIV status or are not in care. Indeed one of the problems of HIV prevention with positives is that about $80 \%$ of the PLWHAs do not know their HIV status as they have never had HIV counselling and testing (HCT) (13). This study was limited to PLWHAs who sought care at one urban centre. PLWHAs who seek care elsewhere (especially in rural areas) may be different from this sample. Furthermore, sexual behaviour was assessed with self-report. Although self-report is commonly used in Uganda and other low income countries for most analyses concerning sexual behaviour (4), there is evidence that self-report may indeed underestimate high risk sexual behaviours (18). 
Implication for interventions: The data suggests that PLWHAs have reproductive needs like desiring more children. However, the most common messages for HIV prevention in this population are sexual abstinence or consistent use of condoms. Yet, evidence from literature implies that a condom which is contraception may not be an appropriate HIV prevention tool PLWHAs who desire children (17). Additional methods of HIV prevention such as using of ART to reduce infectiousness of PLWHAs (19) that could allow conception are needed for prevention with positives. Another method used in high income countries to enable PLWHAs have children is sperm washing a term used to describe the process in which sperms are separated from the seminal fluid by spinning in a centrifuge. The sperms are then used in intrauterine insemination or in vitro fertilization (20). Sperm washing has been developed for couples who wish to have a child, where the male is HIV-positive and the female is HIV-negative. The procedure reduces the risk of HIV transmission to the female partner and subsequently the unborn child because HIV infected material is carried primarily in the seminal fluid rather than in the sperm itself. There is need to evaluate these methods for safety and feasibility among PLWHAs especially in low income settings.

Because PLWHAs who are either ART naïve or ART experienced become pregnant these data suggest that both primary and secondary measures aimed at preventing the spread of HIV from mother to child (PMTCT) services should be given priority. These data also suggest that treatment and control of STIs should deserve utmost attention among PLWHAs as about a quarter of PLWHAs reported symptoms related to an STI within the previous year and STI symptoms make it more likely to transmit and acquire HIV infection. Our data also has further suggestions for targeting of services for positives prevention. High risk sexual behaviour was common among PLWHAs groups mainly those between 31-50 years of age, the married and those who drink alcohol. These variables could be incorporated in risk-reduction counselling to identify PLWHAs who require intense counselling. In order for all these interventions to have impactat the population level, there is need for increasing access to HCT so that the majority of the people are aware of their HIV status and that of their sex partners (13). There is also a strong need for integration of prevention with care $(1,7)$ and to emphasize the correct and consistent use of condoms especially among discordant couples.

\section{ACKNOWLEDGMENTS}

To the research assistants J.Nakintu, R. Tasingika and A. Drasiku for taking part in data collection. Grateful thanks to the Joint Clinical Research Centre for the financial support.

\section{REFERENCES}

1. Collins, C., Morin, S.F., Shriver, M.D. and Coates, T.J. Designing primary prevention for people living with HIV. AIDS Policy Research Center \& Center for AIDS Prevention Studies, Policy Monograph Series. San Francisco, USA. 2000.

2. Crepaz, N. and Marks, G. Towards an understanding of sexual risk behaviour in people living with HIV: A review of social, psychological and medical findings. AIDS. 2002; 16: 135-149.

3. Bunnell, R., Ekwaru, J. P., Solberg, P. et al. Changes in sexual behaviour and risk of HIV transmission after antiretroviral therapy and prevention interventions in rural Uganda. AIDS. 2006; 20: 85-92.

4. Ministry of Health (MOH) [Uganda] and ORCMacro Inc., Uganda HIV / AIDS sero- behavioural survey 2004-2005. Calverton, Maryland, USA: Ministry of Health and ORC Macro. 2006.

5. de Cock, K., Mbori-Ngacha D. A. and Marum, E. Shadow on the continent: public health and HIV / AIDS in Africa in the 21 st century. Lancet. 2002; 360: 67-72.

6. Cates, W. Jr., Chesney, M.A. and Cohen, M.S. Primary HIV infection-a publichealth opportunity. Am. J. Pub. Hlth. 1997; 87: 1928-1930.

7. Kitahata, M.M., Tegger, M.K., Wagner, E.H. and Holmes, K.K. Comprehensive health care for people infected with HIV in developing countries. Brit. Med. J. 2002; 325: 954-957.

8. UNAIDS. From principle to practice: greater involvement of people living with or affected by HIV / AIDS (GIPA). Geneva. 1999.

9. Grosskurth, H., Mosha, F., Todd, J. et al. Impact of improved treatment of sexually transmitted diseases on HIV infection in rural Tanzania: randomized controlled trial. Lancet. 1995; 346: 530-536.

10. Ndembi, N., Lyagoba, F., Nanteza, B. et al. Transmitted antiretroviral drug resistance surveillance among newly HIV type 1-diagnosed women attending an antenatal clinic in Entebbe, Uganda. AIDS Res. Hum. Retro. 2008; 24: 889-895.

11. Palombi, L., Marazzi, M.E., Voetbergc, A. and Magid, N.A. Treatment acceleration program and the experience of the DREAM program in prevention of mother-to-child transmission of HIV. AIDS. 2007; 21 (supp 4): S65-S71.

12. Bateganya, M., Colfax, G., Shafer, L.A. et al. Antiretroviral therapy and sexual behaviour: a comparative study between antiretroviral- naive and -experienced patients at an urban HIV/AIDS care and research center in Kampala, Uganda. AIDS Patient Care STDS. 2005; 19: 760-768.

13. Bunnell, R. and Cherutich, P. Universal HIV testing and counseling in Africa. Lancet. 2008; 371: 21482150.

14. WHO, UNAIDS, \& UNICEF. Towards universal access: scaling up priority HIV / AIDS interventions in the health sector: progress report. April. Geneva. ISBN 978924159539 1. 2007.

15. Kennedy, C., O'Reilly, K., Medley, A. and Sweat, M. The impact of HIV treatment on risk behaviour in developing countries: A systematic review. AIDS Care. 2007; 19: 707-720. 
16. Coldiron, M.E., Stephenson, R., Chomba, E. et al. The relationship between alcohol consumption and unprotected sex among known HIV-discordant couples in Rwanda and Zambia. AIDS Behvr. 2008; 12: 594-603.

17. Nuwaha, F., Faxelid, E. and Hojer, B. Predictors of condom use among patients with sexually transmitted diseases in Uganda. Sex. Transm. Dis. 1999; 9: 491 - 495.

18. Konnings, E., Bantebya, G., Carael, M., Bagenda, D. and Mertens, T. Validating population surveys for the measurement of HIV / STD prevention indicators. AIDS. 2006; 9: 375-382.

19. Montaner, J.S.G., Hogg, R.S., Wood, E. et al. The case for expanding access to highly active anti-retroviral therapy to curb the growth of the HIV epidemic. Lancet. 2006; 368: 531 - 536.

20. Kato, S., Hanabusa, H., Kaneko, S., et al. Complete removal of HIV-I RNA and proviral DNA from semen by the swim-up method: assisted reproduction technique using spermatozoa free from HIV -1. AIDS. 2006; 20: 967-973. 